\title{
Changing Perspectives in Classifications of Hypertensive Retinopathy.
}

\author{
Amit Chopra, Amit Sharma, Surat Singh, Vishwajyoti Bahl \\ Department of Ophthalmology, MM Medical College, Kumarhatti , Solan, India.
}

\begin{abstract}
Hypertensive retinopathy represents the ophthalmic findings of end organ damage secondary to systemic arterial hypertension. The eye is a target organ as well as an established prognostic indicator of systemichypertension. Based on ophthalmoscopically visible alterations, several classifications, the majority of them grading hypertensive fundus changes into four stages have been suggested. The recognition of hypertensive retinopathy is important in cardiovascular risk stratification of hypertensive individuals. The review evaluates the changing perspectives in classification \& prognostic significance of fundal lesions.
\end{abstract}

Keywords: Hypertensive Retinopathy;Classification; Attenuation; Haemorrhage; Hard exudates.

\section{Introduction}

Hypertensive Retinopathy was first described in 1859 by Liebreich ${ }^{(\mathbf{1})}$.Hypertensive Retinopathy consists of retinal vascular changes that are pathologically related to both transient \& persistent microvascular damage from elevated blood pressure. Ocular changes can be the initial finding in an asymptomatic patient with hypertension necessitating primary referral. In other instances, asymptomatic patient may be referred to an ophthalmologist for visual problems caused by hypertensive changes. Elevation of systemic blood pressure causes both focal and generalized constriction of retinal arterioles mediated by autoregulation. These findings are relatively common in long standing hypertension. A prolonged duration of high blood pressure can be associated with a breakdown of inner blood retinal barrier with extravasation of plasma \& RBCs . Retinal haemorrhages, cotton wool spots, intraretinal lipids \& in severe cases, the development of macular star configuration of intraretinal lipid can be seen. Although its name implies only retinal involvement, changes in both choroid \& optic nerve are observed. When choroidal vessels are severely affected by elevated blood pressure, as in acute hypertension, fibrinoid necrosis of choroidal arterioles can cause occlusion of areas of choriocapillaris, with a subsequent breakdown of outer blood retinal barrier. Ocular findings in accelerated hypertension are divided into three distinct categories: hypertensive retinopathy, hypertensive choroidopathy \& hypertensive optic neuropathy.

Ophthalmic findings in acute malignant hypertensive retinopathy include focal arterial narrowing, cotton wool spots, intraretinal transudates, macular edema and retinal haemorrhages. Cystoid macular edema, lipid deposits and arteriolar changes are signs of malignant hypertensive retinopathy ${ }^{(2)}$. Cotton wool spots are fluffy, elevated, tan white areas of retinal opacity occurring within a few disc diameters of optic nerve, caused by occlusion of terminal retinal arterioles. Cotton wool spots typically resolve in 3-6 weeks and are associated with permanent nerve fiber layer loss in the vicinity of the lesion ${ }^{(2)}$. Periarteriolar intraretinal transudates are tan white retinal lesions which resolve without residual retinal damage in 2-3 weeks ${ }^{(3)}$. Macular edema and subretinal fluid are retinal findings related to hypertensive choroidal changes affecting the retinal pigment epithelium, with alterations in the blood retinal barrier. Clinical changes from hypertensive choroidopathy are directly related to the release of endogenous vasoconstrictor agents (eg., angiotensin II, epinephrine, vasopressin) during systemic hypertension. Angiographically, there is delayed, patchy choroida lfilling ${ }^{(2)}$. Gitter et al demonstrated through the use of fluorescein angiography that delay in choroidal filling is followed by late leakage from choroidal vessels into the subretinal space ${ }^{(4)}$. The leakage is enhanced by infarction and damage to the RPE cells or transudation of fluid to subretinal space in response to increase pressure in the choroidal vessels $^{(5)}$. Focal occlusion of the choriocapillaris leads to necrosis \& atrophy of the RPE, forming Elschnig's spots $^{(6)}$. Subretinal fluid accumulates, with the eventual formation of macular edema, a common finding associated with hypertensive choroidopathy ${ }^{(3)}$. Hypertensive optic neuropathy presents clinically as disc edema. This occurs from vasoconstriction of the posterior ciliary arteries supplying the optic nerve head, resulting from the release of angiotensin II and other vasoconstricting agents. In many cases, hypertensive changes are not extensive enough to induce breakdown of either the inner or outer blood retinal barrier. Instead, the chronic effects of hypertension on the retinal vessels become intimately associated with arteriosclerosis changes in the retina characterised by vascular thickening. Hypertensive arteriosclerosis refers to the progressive increase in the elastic \& muscular components of wall of arteriole induced by hypertension. With long standing hypertension, elastic tissue forms multiple concentric layers in intima of the arteriole. The muscular layer can be replaced by collagen fibers \& the intima can be replaced by hyaline thickening. 
With advancing age, however similar changes can develop in the absence of systemic hypertension, these are termed as senile or involutional arteriosclerosis. These changes are accelerated by hypertension. Changes in the retinal circulation in the acute phase of hypertension primarily involve the terminal arterioles. Acute hypertension of any cause can enter into an accelerated or malignant stage, characterized by the fibrinoid necrosis of the arterioles with papilloedema ${ }^{(7)}$. Main retinal arteriolar changes are seen $\&$ recognize as a response to chronic systemic hypertension.

Increased thickening of arteriolar wall caused by arteriosclerotic process causes progressive changes in the appearance of the light reflex from arteriole. Normally the arteriolar wall is invisible, only the column of $\mathrm{RBCs}$ in the lumen is visible as the red line we recognize as the vessel. Reflection of the incident light from the convex surface of the normal arteriolar wall causes a thin line of reflected light to appear in the middle of blood column, the normal light reflex. As the wall becomes thickened, the light reflex loses its brightness \& becomes somewhat broader \& more diffuse in appearance. This is the earliest sign of arteriosclerosis.

With increasing thickness of arteriolar wall \& decreasing lumen, there is further diffusion of the light from the arteriole $\&$ the light reflex takes on the reddish brown hue of the copper wire reflex. As the process continues, there is further thickening of arteriolar wall with associated reduction in the lumen, when the column of blood can no longer be visualized even as a thin line, the arteriole assumes the appearance of silver wire. Arteriosclerotic thickening of the vessel wall also affects the appearance of A-V crossing. The arteriole \& venule share a common adventitial sheath when they cross, with venule lying anterior. Both vascular sclerosis \& perivascular glial cell proliferation contribute to the compression of venule \& constriction of its lumen ,causing appearance of A-V nicking. Seitz attributed the crossing phenomenon to vascular sclerosis \& perivascular glial cell proliferation $\&$ not to venous compression ${ }^{(8)}$. This venous compression varies in severity, from very mild tapering of venous blood column, to more severe tapering, to interruption of visible blood column. The sclerotic changes can also cause deflection of venule as it crosses the arteriole at an acute angle. With increasing sclerotic changes, the venule assumes a more obtuse angle with respect to arteriole at the common crossing \& in severe cases it crosses the arteriole at right angle. Retinal macroaneurysms may also be associated with hypertension. In a reviewof 120 patients with retinal arterial macroaneurysms $67 \%$ had hypertension ${ }^{(9)}$.

The traditional classification of hypertensive retinopathy proposed by Keith et al in 1939 was an attempt to show that severity of hypertension itself was predictive of mortality ${ }^{(10)}$. This classification \& its modifications typically consist of four grades of hypertensive retinopathy with increasing severity. The major limitation of this classification system is the difficulty in distinguishing early hypertensive retinopathy severity (ie grade 1 from grade 2). Several proposals for a new systems have been made ${ }^{(11,12,13)}$. Current literature challenges the prognostic significance of early grade classifications of hypertensive retinopathy by KeithWagener-Barker and Scheie ${ }^{(14,15)}$.

\section{Discussion}

In clinical routine, the widespread classification of Keith-Wagener-Barker 1939 has been a standard for grading fundus changes in patients with arterial hypertension. The poor correlation with severity of hypertension, variation in the onset \& progression of clinical signs \& recognition of optic neuropathy \& choroidopathy as independent entities have prompted some investigators to stress the importance of describing fundal appearance rather than assigning a grade.

Keith Wagener classification is not very helpful as it combines changes of both arteriosclerosis \& hypertension.In 1953, Scheie separately classified arteriosclerotic \& hypertensive changes ${ }^{(8)}$. Both classifications are given in Table 1.

Table 1 Classifications of hypertensive retinopathy: Keith-Wagener-Barker (grade I-IV) was based on the level of severity of the retinal findings; and Scheie (grade 0-4) attempted to quantify the changes of both hypertension and arteriolosclerosis.

\begin{tabular}{|l|l|l|l|}
\hline \multicolumn{2}{|c|}{ Keith-Wagener-Barker } & Scheie \\
\hline Grade & \multicolumn{1}{|c|}{ Features } & Grade & Features \\
\hline & \multicolumn{1}{|c|}{ No changes } \\
\hline I & Mild generalised retinal arteriolar narrowing & 1 & Barely detectable arterial narrowing \\
\hline III & $\begin{array}{l}\text { Definite focal narrowing and arteriovenous } \\
\text { nipping }\end{array}$ & $\begin{array}{l}\text { The above and retinal haemorrhages, exudates } \\
\text { and cotton-wool spots }\end{array}$ & $\begin{array}{l}\text { Obvious arterial narrowing with focal } \\
\text { irregularities plus light reflex changes }\end{array}$ \\
\hline IV & Grade III and papilloedema & $\begin{array}{l}\text { Grade 2 plus copper wiring and retinal } \\
\text { haemorrhages/exudate }\end{array}$ \\
\hline
\end{tabular}

Scheie classification is also in adequate because Pickering pointed out that cotton wool spots are more ominous prognostically than lipid exudation which is another stage 3 change. Also arteriolar changes vary depending upon the previous presence of ageing changes in the vessel i.e involutional sclerosis. Leischman noted that prior 
sclerosis of arteriole seemingly prevented severe arteriolar constriction that occurs as a result of high blood pressure \& reported that it can protect the vessels from severe changes of malignant hypertension. (In young patients with toxaemia of pregnancy or an abrupt elevation of blood pressure related to renal disease, sclerotic changes are not present, pure hypertensive microangiopathy i.e narrowing is observed whereas in chronic hypertension sclerotic changes appear).

Another classification system is the Neubauer Classification system which.is the modification of Keith Wagener system which distinguishes mild (stage 1 - 2) from severe form (stage3-4). Table2

Table 2 Neubauer classification of hypertensive fundus changes

\begin{tabular}{|c|c|}
\hline $\begin{array}{l}\text { Stage I } \\
\text { Optic Disc } \\
\text { Major retinal vessels } \\
\text { Arterioles } \\
\text { Precapillaries } \\
\text { Veins } \\
\text { Venules } \\
\text { Capillaries } \\
\text { Parenchyma }\end{array}$ & $\begin{array}{l}\text { Normal, mild hyperaemia } \\
\text { Normal, barely detectable light reflex changes } \\
\text { Barely detectable arteriolar narrowing } \\
\text { Normal } \\
\text { Mild enlargement (eventually) } \\
\text { Mild tortuosity (eventually) } \\
\text { Visible on the optic disc } \\
\text { Normal }\end{array}$ \\
\hline $\begin{array}{l}\text { Stage II } \\
\text { Optic Disc } \\
\text { Major retinal vessels } \\
\text { Arterioles } \\
\text { Precapillaries } \\
\text { Veins } \\
\text { Venules } \\
\text { Capillaries } \\
\text { Parenchyma }\end{array}$ & $\begin{array}{l}\text { Normal, mild hyperaemia } \\
\text { Obvious increased light reflex changes, obvious narrowing of } \\
\text { vessel wall with focal irregularities } \\
\text { Obvious narrowing of vessel wall with focal or continuous } \\
\text { irregularities, obvious increased light reflex changes } \\
\text { Narrowed, irregular light reflexes } \\
\text { Mild enlargement (eventually) } \\
\text { Mild tortuosity } \\
\text { Few peripapillary loops } \\
\text { Mild haemorrhage, few cotton wool spots (eventually) }\end{array}$ \\
\hline $\begin{array}{l}\text { Stage III } \\
\text { Optic Disc } \\
\text { Major retinal vessels } \\
\text { Arterioles } \\
\text { Precapillaries } \\
\text { Veins } \\
\text { Venules } \\
\text { Capillaries } \\
\text { Parenchyma }\end{array}$ & $\begin{array}{l}\text { Often unsharply bordered, slightly prominent (eventually) } \\
\text { Mild to severe narrowing and focal constriction, irregular light } \\
\text { reflexes } \\
\text { Moderate to severe narrowing, irregular light reflexes } \\
\text { Narrowed, visible alterations of vessel walls } \\
\text { Mild enlargement (eventually) } \\
\text { Mild tortuosity } \\
\text { Ectatic \& grouped haemorrhage, cotton wool spots, } \\
\text { Focal \& hard(circinate) } \\
\text { prethrombosis/thrombosis , capillary bleeding, hadates } \\
\text { exudates }\end{array}$ \\
\hline Stage IV & $\begin{array}{l}\text { Stage III + Papilloedema, peripapillary exudation; exudative retinal } \\
\text { detachment (eventually) }\end{array}$ \\
\hline
\end{tabular}

In 1996 Dodson proposed a classification consisting of only 2 grades: I (arteriolar narrowing, focal constriction \& A-V nicking) and II (haemorrhages \& exudates with or without disc edema) ${ }^{(13)}$. A similar classification has been suggested to encourage the utilisation of the eye as a hypertensive target organ for risk stratification and therapeutic decision making ${ }^{(17)}$. Pache $\mathbf{M}$ in 2002 indicated significant angiographic difference between mild \& severe form of hypertensive retinopathy ie.significant rarefaction of perifoveal capillary bed \& decrease in capillary blood velocity, however unlike in ophthalmoscopy a differentiated division into four stages is not possible $^{(18)}$.

The above explained heterogenous classifications of hypertensive retinopathy, making severity staging a largely arbitrary process, as well as the lack of well defined prognostic value for either systemic outcomes or visual impairment lead to recently proposed Wong classification ( Table 3) based on the concept that an assessment of retinal vascular changes may provide further information for vascular risk stratification in persons with hypertension. Wong formulated 3 grade classification system for hypertensive retinopathy \& suggested approach for patients with each grade 
- Patients with mild HR signs require routine care \& BP control should be based on established guidelines.

- Patients with moderate HR signs (Retinal haemorrhage, cotton wool spots ,hard exudates) may benefit from further assessment of vascular risk eg assessment of cholesterol levels \& if clinically indicated, appropriate risk reduction therapy(cholesterol lowering agents)

- Patients with accelerated hypertension (bilateral disc swelling in presence of moderate hypertensive retinopathy) will continue to need urgent immediate antihypertensive management, including possible administration of IV medications.

Table 3 Classification of hypertensive retinopathy by Wong \& Mitchell

\begin{tabular}{|l|l||l|}
\hline Retinopathy Description & Systemic associations \\
\hline Mild & $\begin{array}{l}\text { One or more of the following signs: generalized arteriolar } \\
\text { narrowing, focal arteriolar narrowing, AV nicking, arteriolar wall } \\
\text { opacity (silver-wiring) }\end{array}$ & $\begin{array}{l}\text { Weak associations with stroke, coronary heart disease } \\
\text { and cardiovascular mortality }\end{array}$ \\
Accelerated & $\begin{array}{l}\text { Mild retinopathy with one or more of the following signs: retinal } \\
\text { haemorrhages (blot, dot or flame-shaped), microaneurysms, } \\
\text { cotton-wool spot, hard exudates } \\
\text { Moderate retinopathy signs plus optic disk swelling; may be } \\
\text { associated with visual loss }\end{array}$ & $\begin{array}{l}\text { Strong association with stroke, congestive heart failure, } \\
\text { renal dysfunction and cardiovascular mortality }\end{array}$ \\
\hline
\end{tabular}

\section{Conclusion}

The original classification system for hypertensive retinopathy was conceived in 1939 by Keith \& colleagues. Since then ,there have been several criticisms of the original system concerning the reproducibility $\&$ the relevance of the system to clinical practice. Subsequently, there have been other classification systems proposed \& focus is towards how does the physician use these data.A clinical assessment of hypertensive retinopathy signs may therefore provide more accurate cardiovascular risk stratification \& potentially guiding treatment strategies.

\section{Bibliography}

[1]. Liebreich R. Ophthalmosk opischer Befundbei Morbus Brightii. Albrecht von Graefes Arch Ophthalmol 1859; :265-268.

[2]. Hayreh SS. Hypertensive fundus changes.In:Guyer DR,ed.Retina-vitreous-macula. Philadelphia:Saunders; 1999:345-71.

[3]. Hayreh SS, Servais GE, Virdi PS. Fundus lesion in malignant hypertension. IV. Focal Intraretinal periarteriolar transudates. Ophthalmology.1986;93:60-73.

[4]. Gitter KA, Houser BP, Sarin LK, Justice J. Toxemia of pregnancy. An angiographic interpretation of fundus changes. Arch Ophthalmol. 1968;80:449-54.

[5]. Fastenberg DM, Fetkenhour CL, Choromolos E, Shoch DE. Choroidal vasculature changes in toxaemia of pregnancy. Am J Ophthalmol. 1980;89:362-68

[6]. Schmidt D, Loffler KU. Elschnig's spots as a sign of severe hypertension. Ophthalmologica.1993;206:24-28.

[7]. Ashton, N and Harry , J: The pathology of cotton wool spots and cystoids bodies in hypertensive retinopathy and other diseases, Trans Ophthalmol R Soc UK 83:91-114,1963.

[8]. Spencer WH. An Atlas and Textbook ( CD-ROM). In : Systemic diseases with retinal involvement : Vascular diseases. Based on : Ophthalmic Pathology. WB Saunders Co:1995.

[9]. Green, WR: Systemic diseases with retinal involvement. In Spencer, WH, ed: Ophthalmic pathology: an atlas and textbook, Philadelphia, 1985, WB Saunders Co, pp1034-1047.

[10]. Keith NM, Wagener HP, Barker NW (1939) Some different types of essential hypertension : their course \& prognosis. Am J Med Sci, 197, 332-43.

[11]. Wong TY, Klein R, Klein BEK, Tielsch JM, Hubbard LD, Nieto FJ (2001) Retinal microvascular abnormalities and their relation to hypertension, cardiovascular diseases \& mortality. Surv Ophthalmol, 46, 59-80.

[12]. Tso M, Jampol LM (1982) Pathophysiology of hypertensive retinopathy. Ophthalmology, 89, 1132-45.

[13]. Dodson PM et al. Hypertensive retinopathy: a review of existing classification systems and a suggestion for a simplified grading system. J Hum Hypertens 1996; 10: 93-98.

[14]. Hayreh SS. Classification of hypertensive changes and their order of appearance. Ophthalmologica 1989; 198: 247-260

[15]. Walsh JB. Hypertensive retinopathy: description, classification and prognosis. Ophthalmology 1982; 8: 1127-1131.

[16]. Scheie, HG: Evaluation of ophthalmoscopic changes of hypertension and arteriolar sclerosis, Arch Ophthalmol49:117, 1953.

[17]. Hyman BN. The eye as a target organ: An updated classification of hypertensive retinopathy. J Clin Hypertens (Greenich) 2000; 2:194-197.

[18]. Pache M, Kube T, Wotf S, Kutschbach P. Do angiographic data support a detailed classification of hypertensive fundus changes? J Hum Hypertens 2002; 16: 405-410. 